\title{
Does Adding Reinforcement of Implementation Intentions Support Behaviour Change?
}

\author{
Adhi Wicaksono \\ School of Computer Science \\ University of Birmingham \\ axw412@cs.bham.ac.uk
}

\author{
Robert J. Hendley \\ School of Computer Science \\ University of Birmingham \\ R.J.Hendley@cs.bham.ac.uk
}

\author{
Russell Beale \\ School of Computer Science \\ University of Birmingham \\ R.Beale@cs.bham.ac.uk
}

\begin{abstract}
It has been suggested that implementation intentions could help people to translate intentions into action and help them form a new habit by repeating their behavioural action consistently. However, implementation intentions remain underused. Previous studies have used reminders to strengthen implementation intentions. However, this could lead to dependency towards the reminders and hinder automaticity. So, in this experiment, we have tried a different approach by conducting a 4week study to investigate the impact of reinforcement on implementation intentions for reporting daily mood. Unlike reminders sent when the action happens, our reinforcements were sent in advance to remind people of their implementation intentions. Our findings suggest that adding reinforcements leads to better compliance but not necessarily increased automaticity. The reinforcements also help people to remember their implementation intentions.
\end{abstract}

Implementation intentions, habit formation, behaviour change intervention.

\section{INTRODUCTION}

Forming new habits allows behaviour change interventions to persist for a prolonged time because when a behaviour has become a habit, the strength of habit will overpower the behavioural intention (Verplanken \& Aarts 1999). Additionally, a habitual behaviour will require less cognitive effort, and it will be performed automatically when a specific situation is encountered (Orbell \& Verplanken 2010; Wood \& Neal 2007; Lally et al. 2010; Lally \& Gardner 2013). The rapid growth of mobile phones has opened a new avenue for developing mobile-based behaviour change intervention. Developers and researchers have worked together to design mobile apps that could support the changes in behaviour via habit formation.

Despite the growing popularity of behaviour change apps that help the formation of new habits, yet only a few of them are built based on the theories of habit and behaviour change. According to Stawarz et al. (2015), the majority of apps that aim to help people form new habits are focused on self-tracking and reminders, neither of which are suitable for supporting habit formation. For example, Streaks, a top-rated habit formation app guides its users to build a new habit by creating a repetitive goal called a "streak". The app does not give guidance to associate the intended goal with existing cues.
Instead, the app uses reminders to keep the consistency of the repeated behaviour by its users.

Although reminders might work for a short period, they could lead to dependency, making an individual rely on the availability of the reminders instead of the actual cues that should trigger the habitual behaviour (Renfree et al. 2016). Reminders also inhibit automaticity as the most important characteristic of habit. When the reminders are removed, people usually forget to act upon their intended goal. Therefore, a better approach is needed to incorporate habit formation into behaviour change apps.

Whereas the majority of behaviour change apps focus on self-tracking and reminders, other strategies such as implementation intentions remain underused. Implementations intentions are a specific action plan which follows a pattern "If situation X happens, then I will do Y" (Gollwitzer 1999). Implementation intentions have been found to be effective in supporting habit formation and to increase the automaticity of behaviour by heightening the accessibility of the cue and strengthening the mental link between the cue and its associated response (Lally \& Gardner 2013; Adriaanse et al. 2011; Stawarz et al. 2015). Implementation intentions are also effective in helping people to achieve their goal (Webb \& Sheeran 2007). 
However, implementation intentions could have a weak effect, especially when the intention to perform the targeted behaviour is not strong enough (Prestwich et al. 2003; Sheeran et al. 2005). Therefore, positive reinforcement is needed in some cases to enhance the impact of implementation intentions.

To our knowledge, research in the area of enhancing implementation intentions remains scarce. Previous studies on this area have used reminders that were sent at due time, reminding people about their intended actions (Prestwich et al. 2009). Therefore, we are interested in trying a different approach and conducted a study to investigate how implementation intentions can be enhanced by adding reinforcement instead of reminders. In this paper, we present our results which suggest that adding reinforcement could strengthen implementation intentions. Participants in our study who received reinforcement had a higher compliance rate compared to the ones who did not receive reinforcement. Additionally, adding reinforcement could help people to recall their implementation intentions by remembering their IF condition as the cue for their intended action. In term of automaticity, we cannot confirm a firm conclusion due to the small sample size.

\section{THE ROLE OF HABIT IN BEHAVIOUR CHANGE}

Habits are repeated behaviours that will be automatically executed as a response towards a particular situation, in which the behaviours are performed consistently (Verplanken \& Aarts 1999). Repeating a behaviour in stable contexts could lead to the formation of a new habit (Lally et al. 2010). However, repetition alone is not enough. To become habitual, behaviour needs to have the elements of automaticity: lack of awareness, unintentionality, uncontrollability, and efficiency (Bargh 1994).

Promoting new habit formation to support behaviour change is more effective than relying on the intentions. A study from Aarts et al. (1997) argues that changing unhealthy habits using the attitude-intentions-behaviour route seems inefficient because intentions no longer guide the behaviour. In another study, Cheung \& Limayem (2005) investigated the relationship between intentions and habits in using information systems. Their findings also suggest that intentions cannot predict the intended behaviour. They found that prior usage of information systems has a more significant role in predicting their use. This finding suggests that intention alone could not be relied upon for successful behaviour change. In reality, it is difficult to maintain the same intentions for prolonged periods because intentions may change over time (Sutton 1998).
The idea of using habit formation to support behaviour change intervention arises as an alternative solution (Tobias 2009). Instead of relying on the intention, habit formation uses subconscious aspect of the mind and could be enhanced by increasing the accessibility of the situational cues. Whereas goal-directed behaviour powered by motivation requires deliberate effort, habits are performed automatically and often unconsciously.

Targeting interventions that help people to perform a behavioural action repeatedly and consistently should lead to habit formation and help maintain the change in behaviour in the a long-term. Lally \& Gardner (2013) argue that forming new habits should follow four stages. Firstly, a decision to take action should be made in the form of intentions. Although intentions are not the only predictor of behavioural action, they still have a strong effect on the action initiation (Fishbein \& Ajzen 1975; Gollwitzer 1993). Secondly, the intentions have to be translated into action. In their study, Webb \& Sheeran (2006) argue that there is an intentionbehaviour gap and this gap could be bridged using self-regulatory or action planning (Schwarzer 2008; Gollwitzer 1999). By creating action planning, someone could also keep his/her intentions and prevent lapse during action initiation. It will also provide a clear pathway from the motivational phase (intentions) to volitional phase (post-intention). Thirdly, when the behaviour is performed, it needs to be repeated. To overcome the challenge of repeating behavioural actions, one should have selfregulatory methods (Abraham \& Michie 2008). And the fourth or final stage, the behavioural response, not only needs to be repeated but it also has to be repeated consistently in the same contexts that could lead to automaticity. Based on the finding from (Lally et al. 2010; Lally \& Gardner 2013), repeating a particular behaviour in a stable context leads to a higher level of automaticity. It means, when the situation is encountered, a behavioural response will be performed automatically. When the behaviour has reached the asymptote of automaticity, the cognitive control to perform such behaviour becomes less needed. Therefore, the long-term change in behaviour should be greater.

\subsection{Implementation Intentions to Support Habit Formation}

Combining behavioural control and current intentions can be effective to enhance habit formation. Oettingen et al. (2001) found that intentions in the form of expectations can improve goal commitment. Ajzen (1991) has also suggested, with the theory of planned behaviour that intention can be a powerful tool that acts as a motivational factor to perform an action. However, the intention to perform such an action can only be achieved if the behaviour being intended is under volitional control (Ajzen 1991). This volitional control can be a specific 
condition whether the action is possible to be done or not.

Whilst the theory of planned behaviour suggests that forming a good intention is needed to achieve a particular goal, Gollwitzer (1999) further investigated the relationship between intention and goal attainment. His findings suggest that successful goal attainment requires a strong commitment from people to get started and do the action until the goal is achieved. He also identifies two reasons for how goal pursuit can be effectively achieved. Firstly, it is important for people to frame their intention in achieving a certain goal. It can be done by setting a specific goal rather than merely a vague goal. Secondly, it is also worth considering that self-regulatory skills in initiating goal-directed behaviour affect the goal attainment.

Compared to the theory of planned behaviour, the theory of goal intentions explains further how intentions can affect goal attainment (Gollwitzer 1999). Goal intentions specify the detailed target of an action, for instance having a goal to walk 10,000 steps each day. The structure of goal intentions usually follows the pattern: "I intend to do X !", In which $X$ is the intended goal (Gollwitzer, 1999). The result of performing goal intentions is that people are more likely to pursue their goals and commit actions to achieve their goals rather than just having the desire to pursue that goal. Therefore, it explains how goal intentions work. It not only sets a specific target for the goal but also commits people to perform such action to realise the goal. In line with the theory of planned behaviour, goal intentions urge people to have a strong intention toward their goal to commit changes in their behaviour.

However, it has also been suggested that having goal intentions is not enough to motivate people into committing to long-term goals. This is due to the gap between people's intentions and their actual behaviour (Sheeran \& Orbell 2000). Moreover, the intentions are not stable for a prolonged period and they may change (Sutton 1998). To overcome this issue, Gollwitzer (1993) came up with a theory called implementation intentions. This theory bridges the gap between intentions and the goal-directed behaviour by providing a clear mechanism from a motivational phase where a decision to achieve a goal is made, to a volitional phase where the detailed plans are made to ensure the goal is achieved. Implementation intentions follow a pattern: "If situation $X$ happens, then I will do action $Y$ ". When an individual follows this pattern, a mental-link will be created between the cue and its associated behaviour. By forming implementation intentions, an individual will commit to perform a particular action that has been planned whenever the situational cue is encountered. Compared to the goal intention, implementation intention furnishes the goal intentions with more specific situational context, including when, where and how the intentions will be performed. When the contextual cues are encountered, they will activate the consciousness of the individual and trigger their mental state to perform such action.

Implementation intentions have been found to have a positive impact on medium-to-large $(d=0.65)$ goal attainment (Gollwitzer \& Sheeran 2006). Additionally, implementation intentions are also shown to increase the rate of goal attainment with evidence on some studies, such as cervical cancer screening (Sheeran \& Orbell 2000), promoting exercise (Prestwich et al. 2003), drivers' compliance with speed limits (Elliott \& Armitage 2006), and fruit intake (Luszczynska et al. 2007).

Despite the promising results, when the intention to perform a behaviour is low, implementation intentions could have a weak effect (Prestwich et al. 2003; Sheeran et al. 2005). Therefore, an implementation intention needs reinforcement to strengthen its effectiveness. One type of reinforcement is to remind the cue as the trigger and the associated behavioural response of the implementation intentions, making the cue and response more salient.

\subsection{Enhancing Implementation Intentions with Reinforcement}

The effect of implementation intentions could be enhanced by adding reminders to the plan. The findings from Prestwich et al. (2009) suggest that using text-messages (SMS) as a reminder could improve the impact of implementation intentions on physical exercising. A further study from Prestwich et al. (2010) also found that adding reminders on implementation intentions had a positive impact on promoting brisk walking. In both studies, the group who received implementation intentions and reminders scored the best results at the end of the study, compared to the other groups (implementation intentions without reminders and control group).

Reminders strengthen two underlying processes of implementation intentions. Firstly, reminders make the cues more salient (Prestwich \& Kellar 2014). Implementation intentions demand the attention from individuals to take action when the specified cues are detected. By adding the reminders, those individuals will have better accessibility towards the cues and increase their awareness of their implementation intentions. Reminders also act as a trigger for the intended action, allowing the intention to be executed immediately. Secondly, reminders also strengthen the mental link between the stimulus (cues) and the behavioural response (Prestwich \& Kellar 2014). When the mental-link between stimulus and response is maintained in a stable context, it will become stronger and there is a higher chance that implementation intentions could make the targeted behaviour become automatic. 
Although previous research that used reminders to support implementation intentions has shown promising results, the mode of delivery for the reminders itself was limited to text messages (SMS) (Prestwich et al. 2009; Prestwich et al. 2010; Stawarz et al. 2015). Additionally, those reminders were sent at the same time as the actual actions. For example, in a study conducted by Stawarz et al. (2015), participants were asked to report their lunch every day, and the participants in the reminders group received a reminder to perform the task at lunchtime. Even though the participants in the reminders group scored best on the adherence of lunch reporting task, they had the lowest score in term of automaticity when performing the behaviour, showing that they depended on the reminder to execute the task. Furthermore, the results of the study have also demonstrated that instead of relying on the situational cues (time for lunch), those groups relied on the reminders to execute their plan (to report their lunch).

Relying on the reminders instead of the actual situation could make the reminder fail in enhancing the impact of implementation intention. Instead of increasing the automaticity towards the defined situations, a reminder could lead to dependency where a person relies on the reminder to execute the intended behaviour, as suggested by Renfree et al. (2016). Moreover, inappropriate reminders via notifications could cause interruptions and adverse effects (Mehrotra 2017). More importantly, if the reminders are often delivered at an inopportune moment, when a person is not able to execute the plan, they could weaken the intention to perform the intended plan. We propose the addition of reinforcement instead of a reminder for supporting implementation intentions. Instead of sending a reminder about one's plan when the actual action is supposed to happen, we will send a reinforcement containing the "if" condition and its associated response in advance (5-6 hours before the actual action). The reinforcement aims to strengthen the association between the cue and its associated behavioural response, as well as to avoid the dependency towards the reinforcement to execute the behavioural action.

\section{METHOD}

Previous research investigating how implementation intentions could be enhanced is limited to adding reminders via SMS and they sent the reminders when the actual action happens (Prestwich et al. 2009; Prestwich et al. 2010; Stawarz 2017). Hence, we applied a different approach in this study by sending the reminders of one's plan as a reinforcement and sent the reinforcement 5-6 hours before the actual action happens. We asked the participants to form an implementation intention of reporting their mood every day for 28 days. Mood report was selected as a task because it is an artificial task, easy to do and it is not a part of any existing routine.

Implementation intention as support for habit formation should be executed automatically and immediately when the cues are encountered, and it is also repeated in a stable context. By adding reinforcement, implementation intentions should be strengthened. So, our hypotheses of this study are:

(i) Participants who receive reinforcement will have a higher compliance compared to participants who do not receive reinforcement.

(ii) Participants who receive reinforcement will have a higher level of automaticity compared to participants who do not receive reinforcement.

\subsection{Participants}

We recruited participants using email, social messaging apps, and meeting them face-to-face without offering a financial incentive. We conducted pre-screening to only recruit participants who used an Android phone. Overall, 58 participants signed up to the study, consisting of 18 males (mean age: 28 years old, $S D=5.61$ ) and 39 females (mean age: 30 years old, $S D=8.92$ ). One participant preferred not to specify the gender.

\subsection{Design}

The study used between-subject design with two different groups:

(i) Control group. Participants in this group were asked to form an implementation intention of reporting their mood every day. They had to select their existing routine as the cue (IF condition). No reinforcement was given to this group.

(ii) Reinforcement group. Participants in this group were asked to form the same implementation intention. They were given an option to choose their routine as the cue. Additionally, we sent them reinforcements that reminded them of their implementation intentions. The reinforcements were sent at lunchtime, way in advance of the actual action to report their mood in the evening.

Two dependent variables were used in this study to measure the differences between the reinforcement and control groups: compliance and automaticity. Compliance was measured by the consistency in reporting the daily mood. Whereas automaticity was measured using the Self-Report Behavioural Automaticity Index (SRBAI) questionnaire. 


\subsection{Materials}

We developed an Android app called Mood Journal for both groups. When opening the app for the first time, the Mood Journal app gave participants stepby-step guidance to create the implementation intentions of reporting their mood in the evening of each day. Participants had to specify their evening routine event as the cue for reporting their mood, for example: when arriving at home, commuting, or after taking a shower. For the reinforcement group, they received reinforcement of their implementation intentions at lunchtime. The reinforcement consisted of the specified routine event that they have selected as a cue (if condition) alongside its associated response (to report their mood). Whereas for the control group, they did not receive any reinforcement. The app recorded their daily mood data and transferred the data securely to our server.

We use Self-Report Behavioural Automaticity Index questionnaire (SRBAI) (Gardner et al. 2012) to measure the automaticity. The SRBAI questionnaire consists of 4 items, asking that "Behaviour $X$ is something ..." ":

- "I do automatically",

- "I do without having to consciously remember",

- "I do without thinking", and

- "I start doing before I realise I'm doing it".

Each of the items has a 7-point Likert scale, and the score of SRBAI is from 4-28 points, where the higher points mean a higher level of automaticity. The SRBAI questionnaire was available online, and the link to access the questionnaire was sent to participants in the second week and fourth week (last day of the study).

To balance the group, we used goal commitment score, measured using HWK scale (Klein et al. 2001). Goal commitment is one of the most prominent moderators of how behaviour could happen, and it can be used to measure the strength of intention.

\subsection{Procedure}

At the start of the study participants were asked to complete a consent form and pre-test questionnaire. Upon completion, participants were selected into two different groups based on their goal commitment score: a control group and reinforcement group. Both groups were asked to install an Android app called Mood Journal. The app guided participants to form a plan (implementation intention) to report their mood. In the plan, participants were asked to choose one existing routine that they usually do in the evening, for example arriving at home or taking a shower. The routine event was used as the cue for reporting mood. So, upon completion of setting implementation intentions, each participant was presented with an if-then plan inside the Mood
Journal app. The format of the plan was "Every evening, after <routine event here>, then I will report my mood".

In the reinforcement group, a daily reinforcement will be sent at lunchtime, consisting of implementation intention (routine event as the cue and reporting mood as the response). For example: "Remember to report your mood after arriving at home in the evening".

Daily mood reports were recorded, as well as the time when the reports were received. On the second week and fourth week $\left(14^{\text {th }}\right.$ and $28^{\text {th }}$ day respectively), a link to access the SRBAl questionnaire was sent to participants. The SRBAI score was used to measure the automaticity in reporting their mood. At the end of the study, participants received debriefing of the study via email.

\section{FINDINGS}

Among 58 participants who signed up to this study, 41 of them (74\%) downloaded and installed the Mood Journal app. We then divide the participants into two different groups: control and reinforcement group. The groups were balanced by participants' commitment, measured with HWK Scale. However, among participants who downloaded the app, only 24 of them reported their mood at least once using the app, where 14 came from the reinforcement group and 10 came from the control group. We only included participants who reported their mood in the analysis.

Over the course of 4 weeks, 241 mood reports were received. We found a stark difference between the two groups with 212 mood reports from participants in the reinforcement group, compared to $29 \mathrm{mood}$ reports within the control group.

\section{Change of compliance}

Compliance was used to measure the consistency of participants in reporting their mood each day. As shown in Figure 1, participants in the reinforcement group had a better compliance rate compared to participants in the control group.

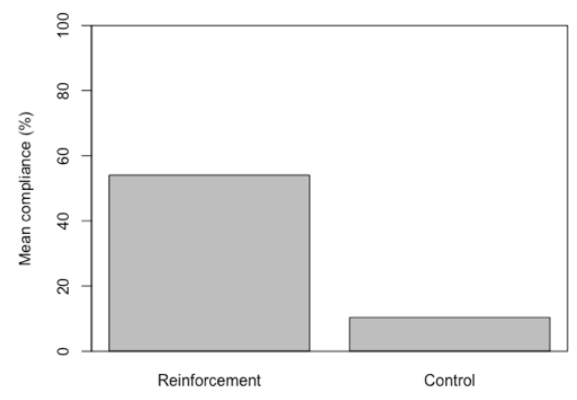

Figure 1: Mean compliance rate of mood reporting task between two groups 
Overall, participants in the reinforcement group had a $54 \%$ compliance rate compared to those in the control group who only had $10 \%$. However, when looking into the changes in compliance, an interesting pattern has emerged. Participants in the control group dropped off significantly after the first day of the study and remained low in compliance throughout the entire study compared to the participants who received reinforcement. Figure 2 shows that even though both groups had a high drop-off rate, participants in the reinforcement group lasted longer than the control group.

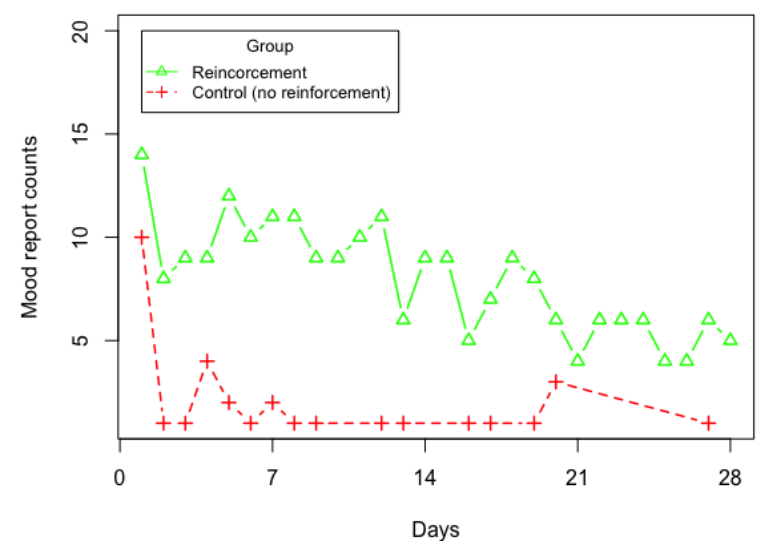

Figure 2: The changes of compliance between two groups, measured using mood report counts

It is important to note that figure 2 shows the number of reports per day. The number of participants who are still active is higher since many will miss completing some reports. Figure 3 shows the number of participants who were still active on the daily basis. Participants were considered to be active if they still sent mood report until a certain day. For example, if a participant sent his/her mood report on the 1st day and went missing, and then reported again on the 7th day, he/she was still considered as active throughout a week.

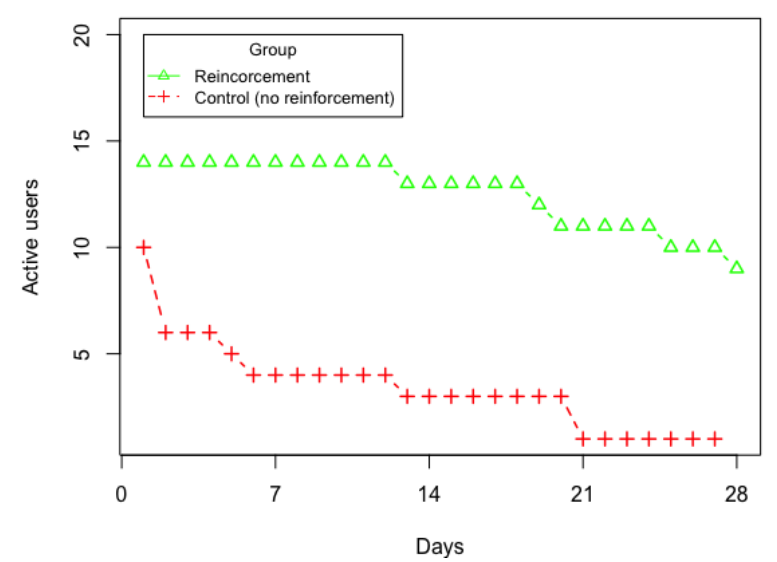

Figure 3: Active users from the beginning until the end of the study

When comparing participants' motivation as measured using HWK scale in the beginning of the study, both groups had a similar average score of their goal commitment (76\%). This means that the participants in both groups had a similar level of commitment and intentions in reporting their mood every day. Figure 2 shows that the majority of participants failed to act upon their intention as shown by the significant drop-off rate, especially in the control group. Whilst in the reinforcement group, the decrease of compliance as measured using mood report counts were slower than the control group. Two weeks after the study ended, eight participants from the reinforcement group were still reported their mood. Conversely, all participants in the control group stopped reporting their mood after the $27^{\text {th }}$ day of the study.

\section{Change of automaticity}

SRBAl was used to measure the strength of automaticity in reporting mood every day. We sent the participants an SRBAI questionnaire at the beginning of the study, second week, and at the end of study (fourth week). We are interested in the changes of the automaticity score between the two groups. On the second week, there were only 6 participants who responded to the SRBAI questionnaire (5 from the reinforcement group, one from the control group).

The same number of participants responded to the SRBAI questionnaire at the end of the study (fourth week). The SRBAI score from the reinforcement group increased from the second week to the fourth week. However, when we looked at the data closely, the two reports from the control group, were from different participants. Therefore, changes of automaticity in the control group from week-2 to week-4 cannot be interpreted. Due to the small sample size, we cannot run an inferential statistical analysis on SRBAI.

\section{Recall of the implementation intentions}

We also investigated how the participants recalled their original cues in their implementation intentions. We sent them a questionnaire asking about their routine as the cue on the second week, and again on the fourth week of the study. There were eight participants who answered the questionnaire either on the second or the fourth week. We then compared the routine events which are recalled by each participant in Table 1.

Overall, only $60 \%$ of participants in the reinforcement group recalled their cues correctly whereas, of the control group, none of them gave the correct answer when remembering their cues. One of the participants in the reinforcement group also mentioned that initially, he/she chose going to $\mathrm{bed} / \mathrm{sleep}$ at night for the routine events but because the app sent the reinforcement at lunchtime, he/she reported his/her mood immediately after receiving the reinforcement. 
Table 1: Recall of routine events as the cue in the implementation intentions to report daily mood

\begin{tabular}{|c|c|l|l|l|}
\hline \multirow{2}{*}{$\begin{array}{c}\text { Participant } \\
\text { ID }\end{array}$} & \multirow{2}{*}{ Group } & \multicolumn{1}{|c|}{ Original cue } & \multicolumn{2}{|c|}{ Recall of the cue } \\
\cline { 3 - 5 } & 1 & Arriving at home & Teaching and its stuff & $\begin{array}{l}\text { Teaching and its } \\
\text { preparation }\end{array}$ \\
\hline P1 & 1 & Arriving at home & Arrived at home & When arrived at home \\
\hline P2 & 2 & Arriving at home & My routine event is study & - \\
\hline P3 & 1 & Going to bed & $\begin{array}{l}\text { I chose night sleep, but my } \\
\text { app keeps ringing on the } \\
\text { middle of afternoon, so I } \\
\text { just report my mood on that } \\
\text { time }\end{array}$ & Night sleep \\
\hline P4 & 1 & Going to bed & Work & - \\
\hline P5 & 2 & Arriving at home & - & Watching movie \\
\hline P6 & 1 & Going to bed & Every morning & $\begin{array}{l}\text { Every morning start of } \\
\text { activity }\end{array}$ \\
\hline P7 & 1 & Commuting & After commuting \\
\hline P8 & & & & \\
\hline
\end{tabular}

\section{DISCUSSION}

Previous studies have suggested that adding plan reminders could enhance implementation intentions (Prestwich \& Kellar 2014). Our results confirm this argument by showing that the participants who receive plan reminders as a reinforcement had better compliance compared to the participants who did not receive a reinforcement. The compliance, as measured by the mood reports per day, has been shown to decay from the beginning towards the end of the study. However, adding a reinforcement has been shown to slow down the decay of compliance. Even after the study ended, there were still eight participants who reported their mood from the reinforcement group. Considering that the mood report is an artificial task that has not been part of our participants' existing routine, this result suggests that adding a reinforcement can indeed be used to promote action initiation.

This study also confirms that intentions alone cannot be relied upon when committing to perform a behavioural action (Scholz et al. 2008). Both groups had a notably high drop-off rate even though they have a good intention to report their mood every day as measured using HWK scale at the beginning of the study. This might also be affected by the type of the behaviour itself. As we mentioned earlier, mood report is an artificial task. Most people might not have an intention to report their mood every day unless they really need it. Thus, their intention to record daily mood may not be strong enough to make the task consistently performed every day. As the theory of planned behaviour suggests, attitude towards the intended predictor still acts as an important predictor for the intention (Fishbein \& Ajzen 1975). However, intention is not sustainable for longer periods of time. The aim of adding reinforcement is to help the participants perform their intended task repeatedly in the stable context, even when their intention is weak.

According to Tobias (2009), reminding someone to perform a behaviour can use three different elements: reminding by events, reminding by executing the behaviour itself, and reminding by situational cues. Existing studies that use reminders focus on situational cues, asking people to perform the intended action when the actual cues were encountered. However, a reminder does not necessarily need to be sent at the actual time when the cues happen because the effect of situational cues does not depend on time (Tobias 2009). Additionally, reminders decay over time and the effect become less significant. Thus, our approach of giving reinforcement 5-6 hours in advance should also have an effect in promoting behaviour as suggested by our findings on the compliance of mood report, without making participants dependant towards the reinforcement. We argue that sending a reminder at the actual time could lead to dependency where people will associate the intended behaviour with the reminder. The study from Renfree et al. (2016) suggests that even though habit formation apps that use reminders could support the repetition of new behaviours, it makes their users become dependent on the reminder to remember performing the behaviour. Instead, we utilise prospective memory by sending the reinforcement of one's plan way in advance, so they can recall their original plan and help them to strengthen their cue-response's link.

According to previous literature, there are two different types of prospective memory: time-based and event-based (Sellen et al. 1997; Brewer et al. 
2017). Time-based prospective memory is a mechanism of remembering to perform a behaviour at a specific time. For example, remember to submit an assignment at $12 \mathrm{pm}$. Different from time-based, event-based prospective memory involves remembering to perform a particular behaviour when a specific situation or cue is encountered. Many activities in our daily live fall into this category. We often have to remember something that is not constrained by time but by other situations. For example, when we pass a gym near our home, we remember to exercise. Event-based prospective memory will help people to remember something by associating the intended behaviour with specific cues. A study from Sellen et al. (1997) found that using event-based cues is better than time-based cues, even though people who use an event-based cue take more time to process the cue. It is related to how the association of cue and its associated response are formed. In implementation intentions, forming a specific if-then plan takes time to make the intended plan be performed consistently. In the beginning, forming implementation intentions requires deliberate effort and sufficient intention to perform the intended behaviour. Otherwise, people will forget about their plan. As our findings have suggested, participants in the control group who formed implementation intentions failed to act upon their plan. Conversely, participants who received reinforcement could execute their plan more consistently.

Accessibility also has an important role in determining behaviour change. According to Tobias (2009), accessibility to perform a behaviour decays over time. He argues that remembering to perform a behaviour becomes more difficult as the time passes. Our results confirm this argument where the compliance of mood report decreases over time. However, adding the reinforcement slows the decay, making people remember their intended task. However, accessibility could also be affected by other factors. In our case, we found that there was a high drop-off rate after the first day of the study. Some of our participants complained about privacy concerning permissions asked by the Mood Journal app including location, mood data, access to phone storage, and internet. Those permissions might become a concern for some participants, and thus it reduces their accessibility and then they decided to stop using the app.

Another interesting finding is the change of automaticity. According to the previous research, repeating a particular behaviour consistently in a stable context could lead to a higher level of automaticity. Although our results show that the automaticity score from the reinforcement group increased from the beginning until the end of the study, in contrast, the automaticity score from the control group decreased significantly after the second week. However, as we mentioned earlier, there was only one participant who responded to the SRBAI questionnaire from the control group each in week 2 and week 4 . So, the result for the control group cannot be interpreted. The small sample size in this study also makes it impossible to run inferential statistics.

In terms of recalling the routine event as a part of the implementation intentions, our results suggest that the participants had a difficulty to remember their routine events as the cue for reporting their mood. This might be due to the reinforcements that were sent at lunchtime (around 12-2pm), whereas their goal of reporting their mood should be done in the evening. Initially, we argue that by sending the reinforcement in advance from the actual action, we can minimise the dependency towards the reinforcement for reporting a mood. Yet, it seems that the participants expected to receive the reinforcement based on the routine event that they chose. For example, when they chose "going to bed" as the cue, they expected to receive a reinforcement about their implementation intentions at night when they are about going to sleep. Our reinforcements were not context-aware, and they were sent at approximately same time every day. This might also be the cause of participants having difficulty in recalling their routine as a cue. Moreover, sending the reinforcement at an inopportune moment might be annoying for some of them, and it might lead to some adverse effects (Mehrotra et al. 2015).

Future work should investigate how different strategies and timing of the reinforcement could affect the implementation intentions itself. In order to minimise the dependency towards the reinforcement, we might be able to phase out the reinforcement if the participants start committing to their intended plan. The reinforcement could also be made context-aware, for example adapting to the situation of an individual.

Additionally, this study only included a small sample. Therefore, future works should involve a higher number of participants to allow inferential statistical analysis. In term of behaviour as the targeted task, we used mood report as an artificial task that has not been a part of existing routine, allowing us to test whether we can enhance implementation intentions on a new behaviour that is not part of the routine. Our findings suggest that adding reinforcement is indeed useful in increasing the compliance of reporting daily mood data as an artificial task. Therefore, we might use the same method on a more meaningful health-behaviour that could be beneficial for the participants such as exercising, drinking more water, or meditation. The different type of behaviour might give a different level of motivation to the participants. So, adding reinforcement for implementation intentions on health-related behaviour might lead to a higher level 
of compliance because people are more motivated to do so.

\section{CONCLUSION}

This paper investigates how implementation intentions can be enhanced by adding reinforcement. Unlike other studies that tried to enhance implementation intentions by sending reminders at around the actual times of the intended action, we tried a different approach by sending the plan reminder as a reinforcement way in advance before the actual action should happen. We tested whether the reinforcement gave a positive impact on the implementation intentions or not. We measured the changes of compliance and automaticity of a daily mood report task.

Even though our initial findings suggest that giving reinforcement improves compliance, it is difficult to draw reliable conclusions due to the small sample size. A similar finding was found for the automaticity where there was only one participant from the control group who answered the SRBAl questionnaire in both of week-2 and week-4 of the study. The type of behaviour and reinforcement might be the factors. Mood report as the task in this study is an artificial task that does not belong to the participants' existing routine. Therefore, it might not be a relevant or important task for some of them. Future work is needed to investigate how using a different type of behaviour (for example exercising), and different type of reinforcement (for example using context-aware reinforcement) could have an impact on implementation intentions to help the formation of a new habit.

Although intention could not be used to predict a behaviour in the long-term, it still plays an important role in determining the behaviour. In our findings, participants failed to act upon their good intentions, especially in the control group. Choosing a more meaningful behaviour could lead to a better result because people would have a stronger intention to do so. Additionally, having a behaviour that requires more intention will help people committing to their plan.

Implementation intentions require a strong commitment from an individual who wants to use them. Additionally, when people start forming an implementation intention, they still need reinforcement because they tend to forget about their plan, as our findings have suggested. People had a difficulty in recalling their plan. Therefore, reinforcement of one's plan is needed to strengthen their implementation intention. We used reinforcement that utilises prospective memory, more specifically event-based memory where we help participants to associate the mood report task with their existing routine as a cue. As the reinforcements were sent way in advance from the actual action, we hoped that the participants could remember their implementation intentions and did not rely on the reinforcement to report their mood. Participants who received reinforcement had indeed a better recalling performance compared to the others without reinforcement. Unfortunately, we cannot measure the dependency towards the reinforcement because of the small sample in our study.

\section{REFERENCES}

Aarts, H., Paulussen, T. \& Schaalma, H., 1997. Physical exercise habit: on the conceptualization and formation of habitual health behaviours. Health education research, 12(3), pp.363-374.

Abraham, C. \& Michie, S., 2008. A taxonomy of behavior change techniques used in interventions. Health Psychology, 27(3), pp.379-387. Available at:

http://doi.apa.org/getdoi.cfm?doi=10.1037/027 8-6133.27.3.379.

Adriaanse, M.A. et al., 2011. Breaking Habits With Implementation Intentions: A Test of Underlying Processes. Personality and Social Psychology Bulletin, 37(4), pp.502-513. Available at:

http://psp.sagepub.com/content/37/4/502.abst ract.

Ajzen, I., 1991. The theory of planned behavior. Organizational behavior and human decision processes, 50(2), pp.179-211.

Bargh, J.A., 1994. The four horsemen of automaticity: Awareness, intention, efficiency, and control in social cognition. In R. Wyer \& T. Srull, eds. Handbook of Social Cognition. Lawrence Erlbaum.

Brewer, R.N., Morris, M.R. \& Lindley, S.E., 2017. How to Remember What to Remember: Exploring Possibilities for Digital Reminder Systems. Proc. ACM Interact. Mob. Wearable Ubiquitous Technol., 1(3), p.38:1--38:20. Available at: http://doi.acm.org/10.1145/3130903.

Cheung, C. \& Limayem, M., 2005. The role of habit in information systems continuance: examining the evolving relationship between intention and usage. ICIS 2005 Proceedings, p.39.

Elliott, M. a \& Armitage, C.J., 2006. Effects of implementation intentions on the self-reported frequency of drivers' compliance with speed limits. Journal of experimental psychology. Applied, 12(2), pp.108-117.

Fishbein, M.E. \& Ajzen, I., 1975. Belief, Attitude, Intention, and Behavior: An Introduction to Theory and Research, Reading, MA: AddisonWesley. 
Gardner, B. et al., 2012. Towards Parsimony in Habit Measurement: Testing the Convergent and Predictive Validity of an Automaticity Subscale of the Self-Report Habit Index. The International Journal of Behavioral Nutrition and Physical Activity, 9, p.102. Available at: http://www.pubmedcentral.nih.gov/articlerend er.fcgi?artid=3552971\&tool=pmcentrez\&rende rtype $=$ abstract.

Gollwitzer, P.M., 1993. Goal achievement: The role of intentions. European review of social psychology, 4(1), pp.141-185.

Gollwitzer, P.M., 1999. Implementation intentions: Strong effects of simple plans. American Psychologist, 54(7), pp.493-503.

Gollwitzer, P.M. \& Sheeran, P., 2006. Implementation intentions and goal achievement: A meta-analysis of effects and processes. Advances in experimental social psychology, 38, pp.69-119.

Klein, H.J. et al., 2001. The Assessment of Goal Commitment: A Measurement Model MetaAnalysis. Organizational Behavior and Human Decision Processes, 85(1), pp.32-55. Available at: http://linkinghub.elsevier.com/retrieve/pii/S074 9597800929315 [Accessed July 20, 2017].

Lally, P. et al., 2010. How are habits formed: Modelling habit formation in the real world. European Journal of Social Psychology, 40(6), pp.998-1009.

Lally, P. \& Gardner, B., 2013. Promoting habit formation. Health Psychology Review, 7(sup1), pp.S137-S158. Available at: http://www.tandfonline.com/doi/abs/10.1080/1 7437199.2011.603640 [Accessed March 31, 2017].

Luszczynska, A., Tryburcy, M. \& Schwarzer, R., 2007. Improving fruit and vegetable consumption: a self-efficacy intervention compared with a combined self-efficacy and planning intervention. Health Education Research, 22(5), pp.630-638. Available at: http://her.oxfordjournals.org/content/22/5/630. abstract.

Mehrotra, A., 2017. A framework for intelligent mobile notifications. University of Birmingham. Available at: http://etheses.bham.ac.uk/7440/1/Mehrotra17 PhD.pdf.

Mehrotra, A. et al., 2015. Designing Content-driven Intelligent Notification Mechanisms for Mobile Applications. In Proceedings of the 2015 ACM International Joint Conference on Pervasive and Ubiquitous Computing. UbiComp '15. New York, NY, USA: ACM, pp. 813-824. Available at: http://doi.acm.org/10.1145/2750858.2807544.

Oettingen, G., Pak, H. \& Schnetter, K., 2001. SelfRegulation of Goal Setting: Turning Free Fantasies About the Future Into Binding
Goals. Journal of Personality and Social Psychology, 80(5), pp.736-753.

Orbell, S. \& Verplanken, B., 2010. The automatic component of habit in health behavior: habit as cue-contingent automaticity. Health Psychology, 29(4), p.374.

Prestwich, A. \& Kellar, I., 2014. How can the impact of implementation intentions as a behaviour change intervention be improved? Revue Européenne de Psychologie Appliquée/European Review of Applied Psychology, 64(1), pp.35-41. Available at: http://www.sciencedirect.com/science/article/p ii/S1162908810000186 [Accessed June 23, 2015].

Prestwich, A., Lawton, R. \& Conner, M., 2003. The use of implementation intentions and the decision balance sheet in promoting exercise behaviour. Psychology \& Health, 18(6), pp.707-721. Available at: http://dx.doi.org/10.1080/0887044031000159 4493.

Prestwich, A., Perugini, M. \& Hurling, R., 2010. Can implementation intentions and text messages promote brisk walking? A randomized trial. Health Psychology, 29(1), pp.40-49.

Available at:

http://doi.apa.org/getdoi.cfm?doi=10.1037/a00 16993.

Prestwich, A., Perugini, M. \& Hurling, R., 2009. Can the effects of implementation intentions on exercise be enhanced using text messages? Psychology \& health, 24(6), pp.677-687.

Renfree, I. et al., 2016. Don't Kick the Habit: The Role of Dependency in Habit Formation Apps. In Proceedings of the $2016 \mathrm{CHI}$ Conference Extended Abstracts on Human Factors in Computing Systems - CHI EA '16. pp. 29322939. Available at: http://dl.acm.org/citation.cfm?doid=2851581.2 892495.

Scholz, U. et al., 2008. Beyond behavioural intentions: Planning mediates between intentions and physical activity. British journal of health psychology, 13(3), pp.479-494.

Schwarzer, R., 2008. Modeling health behavior change: How to predict and modify the adoption and maintenance of health behaviors. Applied Psychology-An International Review, 57(1), pp.1-29.

Sellen, A.J. et al., 1997. What Brings Intentions to Mind? An in Situ Study of Prospective Memory. Memory, 5(4), pp.483-507.

Sheeran, P. \& Orbell, S., 2000. Using implementation intentions to increase attendance for cervical cancer screening. Health psychology: official journal of the Division of Health Psychology, American Psychological Association, 19(3), pp.283289. 
Sheeran, P., Webb, T.L. \& Gollwitzer, P.M., 2005.

The Interplay Between Goal Intentions and Implementation Intentions. Personality and Social Psychology Bulletin, 31(1), pp.87-98. Available at: http://psp.sagepub.com/content/31/1/87.abstr act.

Stawarz, K., Cox, A.L. \& Blandford, A., 2015.

Beyond Self-Tracking and Reminders:

Designing Smartphone Apps That Support Habit Formation. In Proceedings of the 33rd Annual ACM Conference on Human Factors in Computing Systems. CHI '15. New York, NY, USA: ACM, pp. 2653-2662. Available at: http://doi.acm.org/10.1145/2702123.2702230.

Stawarz, K.M., 2017. Towards better medication adherence apps: Preventing forgetfulness by facilitating the formation of routine-based remembering strategies. University College London.

Sutton, S., 1998. Predicting and explaining intentions and behavior: How well are we doing? Journal of Applied Social Psychology, 28(15), pp.1317-1338. Available at:

http://onlinelibrary.wiley.com/doi/10.1111/j.15 59-1816.1998.tb01679.x/abstract.

Tobias, R., 2009. Changing behavior by memory aids: A social psychological model of prospective memory and habit development tested with dynamic field data. Psychological Review, 116(August), pp.408-438. Available at: http://psycnet.apa.orgjournals/rev/116/2/408.

Verplanken, B. \& Aarts, H., 1999. Habit, Attitude, and Planned Behaviour: Is Habit an Empty Construct or an Interesting Case of Goaldirected Automaticity? European Review of Social Psychology, 10(September), pp.101134.

Webb, T.L. \& Sheeran, P., 2006. Does changing behavioral intentions engender behavior change? A meta-analysis of the experimental evidence. Psychological bulletin, 132(2), p.249.

Webb, T.L. \& Sheeran, P., 2007. How do implementation intentions promote goal attainment? A test of component processes. Journal of Experimental Social Psychology, 43(2), pp.295-302.

Wood, W. \& Neal, D.T., 2007. A new look at habits and the habit-goal interface. PSYCHOLOGICAL REVIEW, 114(4), pp.843863. 